\title{
Predictive Washout Filter Design for VR-based Motion Simulator
}

\author{
Su-Chiun Wang \\ Department of Electrical Engineering \\ National Taiwan University \\ Taipei, Taiwan, ROC \\ r91921007@ntu.edu.tw
}

\begin{abstract}
Modern simulators normally consist of hydraulic motion platform, computer visual systems, forcefeedback systems, and sound systems such that the operator is provided realistic motion cues given the state of the vehicle. Owing to the limited motion workspace of typical motion platform, filtering is required between the computer vehicle motion and the desired simulator motion trajectory. These filters are commonly referred 10 as washout filter. Unlike most of the washout algorithms which focus on how to tune the filter parameters wistly or flexibly, this paper proposes a method which can divectly manages the simulator trajectory by moving it freely in the workspace without the sensation of operator. By this approach, simulation results show a smaller workspace required under the same filter parameters. In other words, more aggressive filter parameters can be chosen under the same constraint of limited workspace, which indirectly implies that a more realistic simulator performance can be attained.
\end{abstract}

Keywords: Washout filter, simulator, predictive, virtual reality.

\section{Introduction}

The washout filters, whose name originates from the fact that one of their functions is to "wash out" the position of the simulator back to its neutral point, remain the dominate source of poor fidelity in the motion cues, and even a small refinement in this area can yield a significant improvement in motion realism [1].

Many different schemes of washout filter have been proposed in the last three decades [2]. Classical washout filter were the first to be developed which is composed of linear low-pass and high-pass filters and characterized by its simplicity and ease of adjustment. But owing to the inflexibility of the classical washout filter, many washout algorithms with the parameter self-tuning mechanism have been proposed to solve this problem. Adaptive schemis tune the filter gain in real time to minimize a cost function using steepest descent techniques [1]. The optimal control algorithm is characterized by a systematic combination of linear filters that is determined by an off-line clesign algorithm which produce the optimal form, order, and

\author{
Li-Chen Fu, Fellow, IEEE \\ Department of Electrical Engineering \\ National Taiwan University \\ Taipei, Taiwan, ROC \\ lichen@ntu.edu.tw
}

characteristics for the filters given the underlying assumptions. Recently, an auto-tuning washout filter based on the adaptive algorithm and neuro-fuzzy network is proposed [3]. Beside, considering the case where the motion platform could be of limited bandwidth, low-driven power, or under poor position control, the robust control algorithm which adopts the closed-loop form with feedbacks of the platform position, attitude, and even accelerometer measurement is proposed [4].

This paper is organized as follows. Section 2 reviews the classical washout filter. Then, the predictive washout filter is proposed in section 3 and the related simulation and experiment results are shown in section 4 and 5 . And, the conclusion is made in section 6 .

\section{Classical Washout Filter}

The classical algorithm $[1,6]$ is the most widely used for commercial simulators. It is characterized by the empirically determined combination of linear high- and low-pass filters whose break frequencies and damping ratios can be adjusted off-line by trial and error. A flowchart of the classical algorithm implemented in the present work is shown in Fig 1.

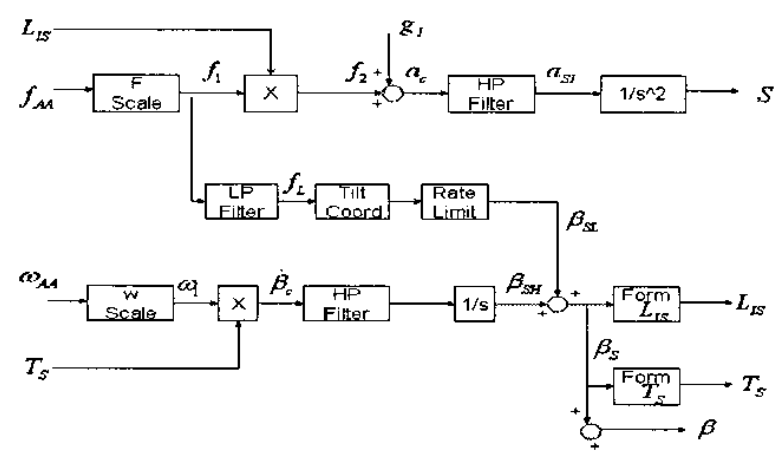

Figure 1. Classical algorithm

The inputs to the algorithm are three vehicle specific forces in body axes, defined as $f_{A A}=a_{A A}-g_{A}$, and the three vehicle angular rates. The vehicle specific forces are highpass filtered to yield the simulator translational accelerations under the assumption that low-frequency specific forces would tend to drive the motion base to its 
limits. The longitudinal and lateral specific forces are also passed through low-pass filters, scaled, and rate-limited to produce pitch and roll tilt angles, respectively. The purpose of this "tilt-coordination" mechanism is to orient the gravity vector in the simulator in the same way with regard to the operator as the low-frequency force in the vehicle, thus allowing sustained vehicle accelerations to be simulated. The vehicle angular motion is high-pass filtered to yield the high-frequency component of simulator angular motion, whereas the tilt mechanism described above supplies the low-frequency component.

The principal disadvantage of this scheme is that because of their fixed parameters, classical filters must be designed for worst-case maneuvers and often yield minimal motion under gentler maneuvering.

Owing to this condition, various algorithms have been proposed. For example, classical washouts are made up of an empirically determined combination of filters. However, unlike the classical theme, some of the filter parameters are systematically varied for better performance and workspace usage.

But no matter what algorithm is used, there exists a trade-off between parameter tuning and workspace usage. The more aggressive the parameters are chosen, the more workspace is needed. That is, we propose a method which does not focus on parameters tuning algorithm, but manage the workspace directly. Such method is compactable with any washout filter developed, and extends the range of which the parameters can be selected.

\section{Predictive Washout Filter Design}

The main idea of this algorithm is to directly manage the trajectory of motion platform under the premise of not interfering the original perception of the operator. To make the movement "senseless" to the operator, the acceleration must be under the threshold value of human perception (Table 1).

Table 2. Sensation threshold value of Otolith [5]

\begin{tabular}{|c|c|c|c|}
\hline & Surge & Sway & Heave \\
\hline$d_{T H}\left(\mathrm{~m} / \mathrm{sec}^{2}\right)$ & 0.17 & 0.17 & 0.28 \\
\hline
\end{tabular}

If we move the platform only in translational directions, the induced acceleration on body-axis and inertial-axis will be exactly the same. Such motion will be slow and inefficient under the constraint of being "senseless".

We propose a new method which treats the operator as an inverted pendulum. The control goal of the inverted pendulum is to move the pendulum with attitude change as small as possible.

For the inverted pendulum, the linkage between car and pendulum is ball joint. Actually, there isn't any joint between operator and his seat. However, if the platform tilts the same angle as that of the pendulum, the stability problem will be the same as that shown in Fig. 2.
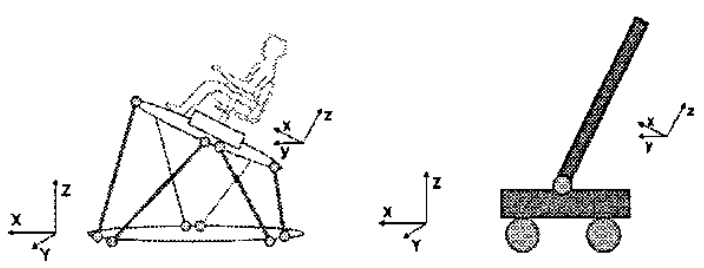

Figure 2. Schematics of senseless manoeuvre

This algorithm itself is not a complete washout filter. It is a function which provides a method to move the platform directly and senselessly. In other words, by a simple superposition of the output signal of this method and that of washout filter, the performance can be improved by a better usage of the platform workspace. Figure 4 is an example of the combination of predictive function and classical washout filter. Attention is drawn to the following points in Fig.3.

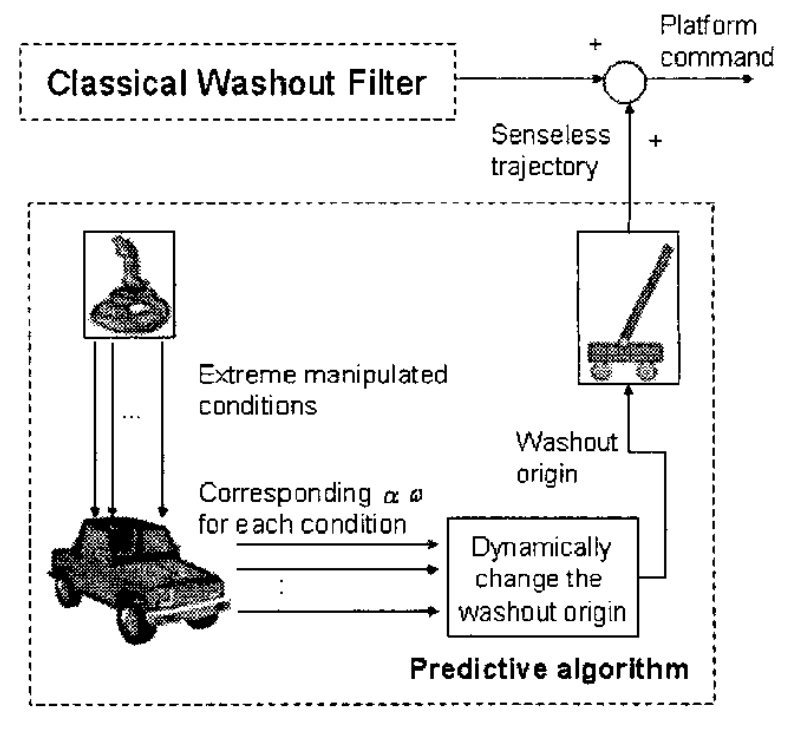

Figure 3. Schematics of predictive algorithm

The predictive function block generates the desired trajectory according to the vehicle dynamics, condition of manipulator, and foreseeable change of environment. The "predictive" means we can predict the movement of the vehicle from the operation conditions stated above and move the platform to a place in advance to keep the workspace large enough for future usage. 
Because the motion generated by predictive furction is senseless, the motion generated by classical washout filter will not be contaminated.

\section{Simulations}

This section comprises two parts. First, we demonstrate the senseless maneuver feature of predictive algorithm. Second, we construct a car dynamic model to verify the effectiveness of the predictive algorithm. Both the classical washout filter with and without predictive algorithm are used to generate the motion in throttle-pulse maneuvering.

\subsection{Senseless maneuvering}

Figure 4 demonstrates the simulation result of senseless maneuvering. The dot line is the acceleration on the inertial coordinate frame, and the solid line is the acceleration on the body coordinate frame, which is relative to the operator's sensation. The results show that under such algorithm, the acceleration sensed by the operator is much smaller than that of the platform really does. Especially when it is under the threshold value, the maneuver will become senseless.

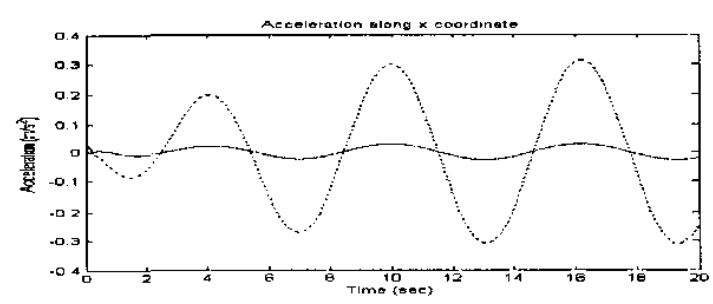

Figure 4. Senseless maneuvering

\subsection{Throttle-pulse maneuvering}

Throttle-pulse maneuver changes the operation state between sudden throttling to the maximum and braking to maximum for every 5 seconds. Fig. 5 shows the simtlation results

For sustained specific force input in sway or surge, the simulator will achieve a steady-state pitch or roll angle because of tilt-coordination. If the input ends abruptly, then the high-pass specific force response will initially cancel out the specific force associated with the tilt, but only for a brief time because the restricted simulator displacement prohibits translational acceleration of the simulator. If the tilt is removed quickly, then false cue of the tiltcoordination angular rate will occur. The severity of this false cue can be alleviated by parameters reduction. However, such tuning will increase the platform displacement as well. (Fig. 5b)

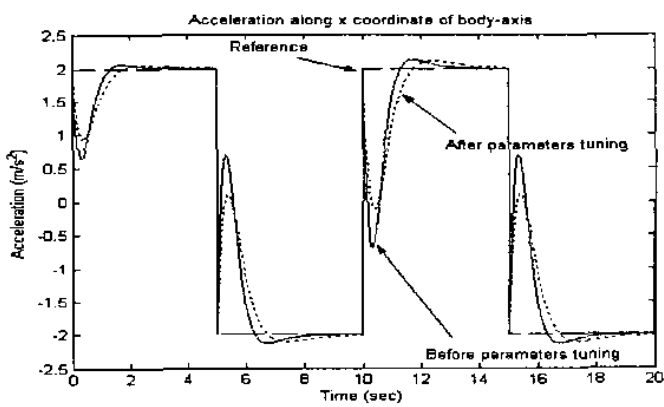

(a)
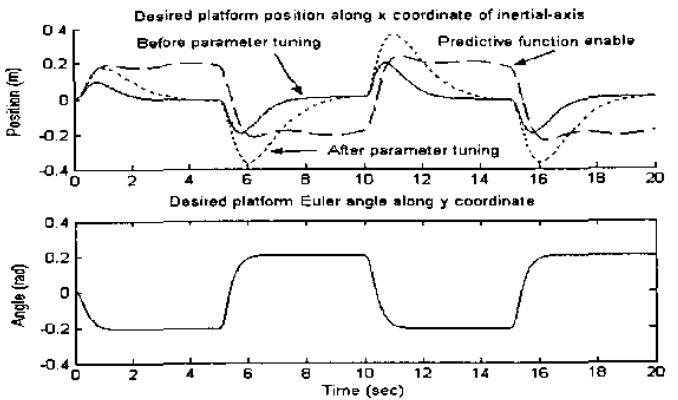

Figure 5. Simulation results

To solve this problem, we use the predictive algorithm. For the predictive mechanism here, we only consider the throttle position to keep it simple. The story is that: at the first 5 seconds, the throttle is driven to the maximum. In case of classical washout filter, platform will return to neutral translational position aftermath (Fig. 5b). On the other hand, judging from the throttle position, we know the car need more room for deceleration than for acceleration. That is, instead of returning to the neutral position, predictive algorithm moves the platform to $0.2 \mathrm{~m}$ of $x$ coordinate (Fig. $5 b$ ). This action is taken to reserve the workspace for the usage of possible future deceleration, and vice versa.

\section{Experiments}
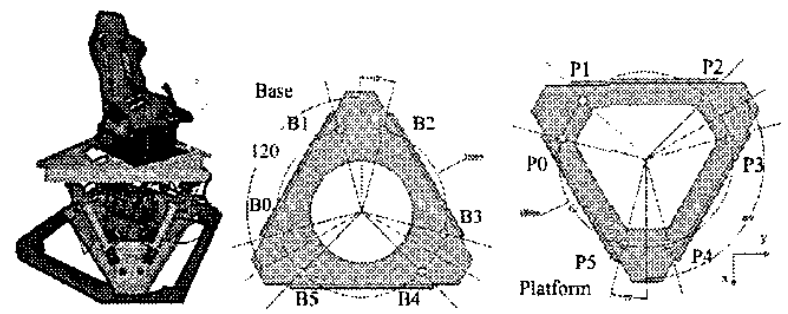

Figure 6. Schematic diagram of Stewart platform

We have built a simulator environment in the Advanced Control Laboratory (ACL) in the Department of Electrical Engineering at the National Taiwan University. The Stewart platform (Fig. 6) which has an accelerometer 
and a tilt sensor attached on it is used for the experiments in this section..

The experiments are divided into two parts in this section. In the first part, the effectiveness of senseless maneuvering is verified through the experiments. Then, the simulation result about the predictive washout filter done in the previous section is verified in the second part.

\subsection{Senseless Maneuvering}

Figure 7 shows the experimental results with and without using the senseless maneuvering algorithm when tracking a sine wave trajectory along $\mathrm{x}$ axis in the platform task space. Fig. $7(\mathrm{~b})$ is the reference trajectory along $\mathrm{x}$ axis. If the senseless maneuvering function is enabled. There will be a corresponding rotation angle according to the senseless maneuvering algorithm (Fig. 7(c)). If the senseless maneuvering function is disabled. No rotation will be made. The relative accelerometer measurements under 2 cases stated above are plotted in Fig. 7(a) for comparison.

In Fig. 7(a), the accelerometer reading with senseless maneuvering algorithm is under the threshold of sensation human. That is, such movement will not be detected by the driver. On the contrary, this is not the case for the accelerometer reading without senseless maneuvering algorithm. In other words, for tracking the same trajectory in the platform task space, the senseless maneuvering algorithm can effectively attain the goal to be "senseless".
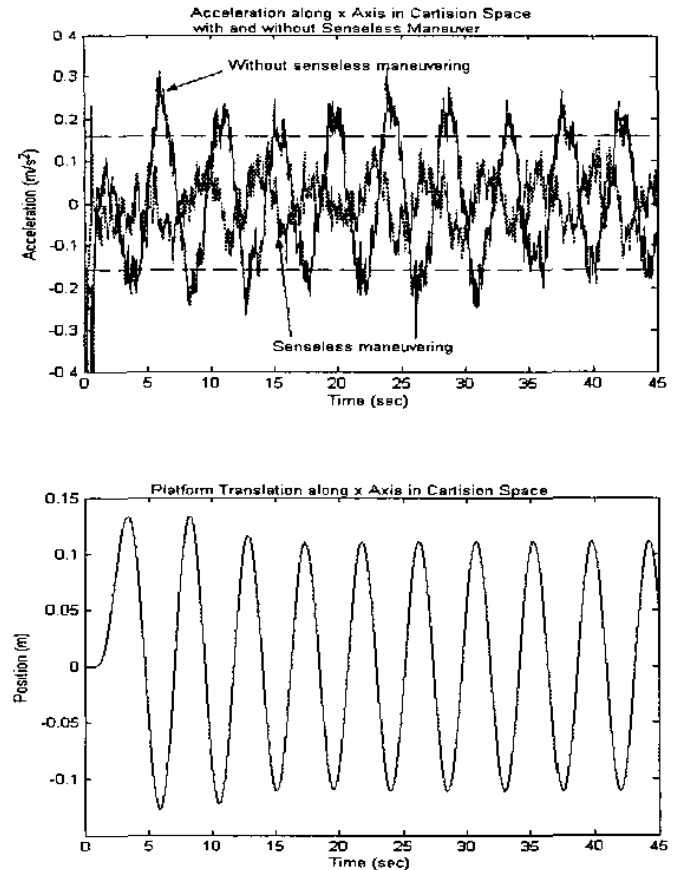

(b)

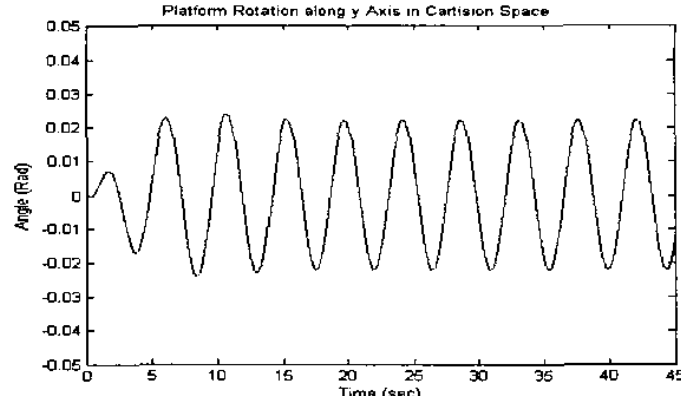

(c)

Figure 7. The experiment result of senseless maneuvering of the Stewart platform for tracking a sine wave trajectory along $\mathrm{x}$ axis in the platform task space

Figure 8 shows the experimental results with and without using the senseless maneuvering algorithm when tracking a step trajectory along $\mathrm{x}$ axis in the platform task space.

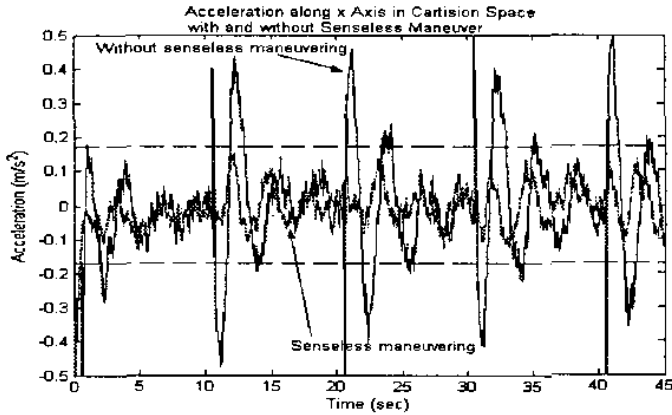

(a)

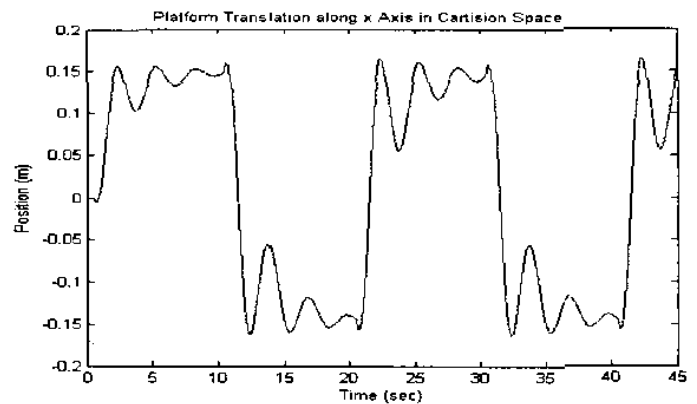

(b)

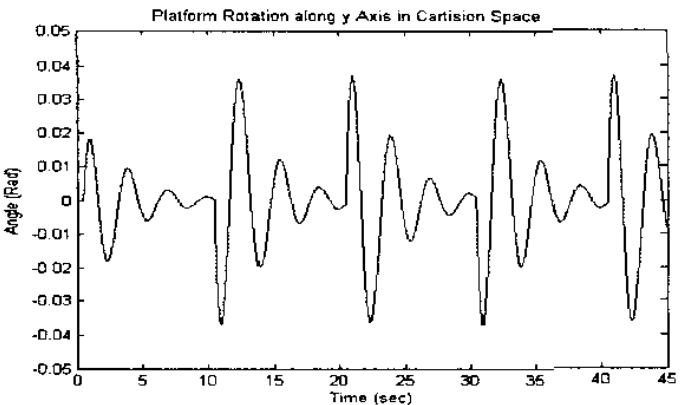

(c)

Figure 8. The experiment result of senseless maneuvering of the Stewart platform for tracking a step trajectory along $\mathrm{x}$ axis in the platform task space 


\subsection{Throttle-pulse maneuvering}

This experiment is done at the same condition as the simulation of throttle-pulse maneuvering in section 4 . The experimental results (Fig.9) are conformed to the simulation results. That is, the feasibility of predictive washout filter design is verified.
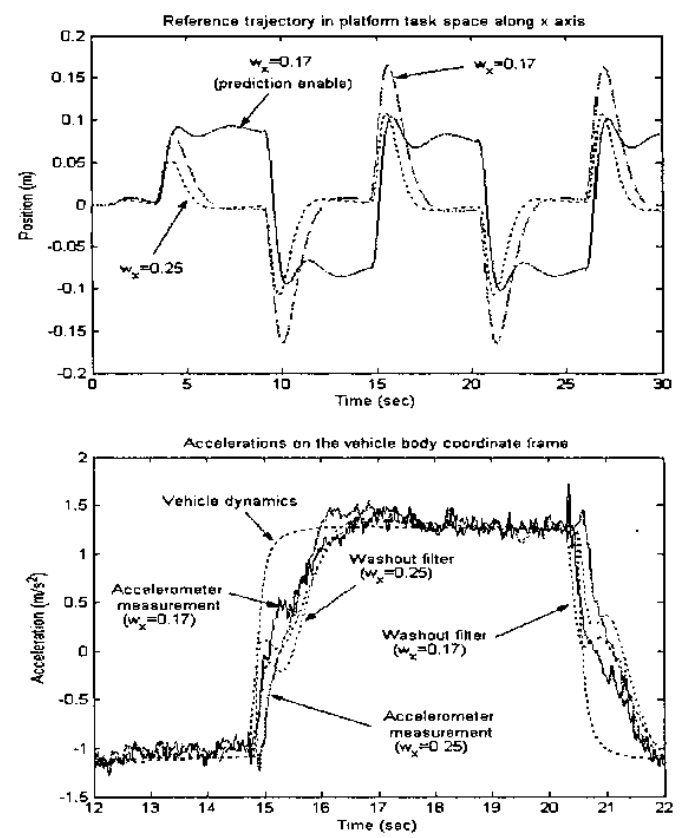

(a)

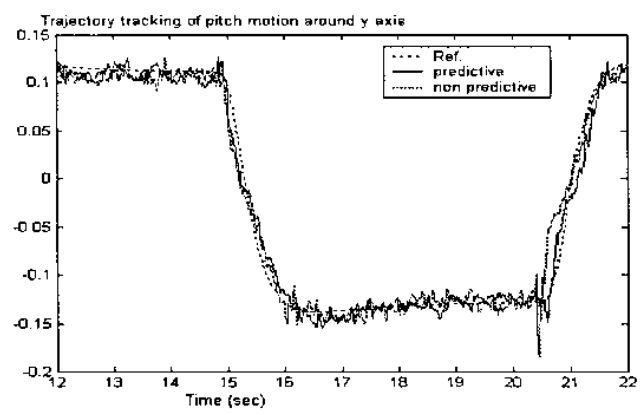

(b)

Figure 9. Experiment results of throttle-pulse maneuvering

\section{Conclusions}

A new method has been developed, which does not focus on parameter tuning, but directly manage the platform workspace. Such method is not a substitute of the current washout filter algorithm. Instead, it is compatible with any existing algorithm to attain a better perforraance. The feasibility of such algorithm is verified through the simulations and experiments. And, the results show that a better performance can be attained.

\section{References}

[1] M.A. Nahon, L.D. Reid and J. Kirdeikis, "Adaptive Simulator Motion Software with Supervisory Corttrol,",
Journal of Guidance, Control, and Dynamics, vol.15, no.2 pp.376-383 1992

[2] F. Barbagli, D. Ferrazzin, C.A. Avizzano and M. Bergamasco, "Washout Filter Design for a Motorcycle Simulator,", IEEE International Conference on Systems, Man, and Cybernetics, vol.2 pp.995-1000 2000

[3] J.S. Yan and C.T. Lin, "Auto-tuning Washout Filter and its Application to VR- based Ship Dynamic Simulation System" Master Thesis of NCTU 2000

[4] M. Idan and M.A. Nahon, "Off-Line Comparison of Classical and Robust Flight Simulator Morion Control,", Journal of Guidance, Control, and Dynamics, vol.22, no.5 1999

[5] M.K. Park, M.C Lee, K.S. Yoo, K. Son, W.S. Yoo, and M.C. Han, "Development of the PNU Vehicle Driving Simulator and Its Performance Evaluation,", Proceeding of IEEE International Conference on Robotics and Automation, pp.2325-2330 2001

[6] M.A. Nahon and L.D. Reid, "Simulator Motion-Drive Algorithm: A Designer's Perspective, ", Journal of Guidance, Control, and Dynamics, vol.13, no.2 pp.356-362 1990 